\title{
Kein Platz für den Produktbezug?
}

\section{Die derzeit gültige europäische EG-Öko-Audit-Verordnung isł auf den einzelnen Standort eines Unternehmens ausgerichtet. Damit grenzt sie sich nicht nur von einem möglichen Organisationsbezug der ISO 14001, sondern auch von einem Produktbezug ab. Ein solcher würde eine Berücksichtigung des Lebensweges eines Produktes von der Wiege bis zur Bahre erfordern und müBte damit nicht nur standortübergreifend, sondern sogar organisationsübergreifend sein.}

Von Mario Schmidt

0 tandort- oder Produktbezug können als verschiedene Perspektiven bzw. Abgrenzungen ein- und desselben Systems - nämlich des Produktionssystems - verstanden werden. Je nach Wahl werden dabei andere Aspekte beleuchtet bzw. unterschiedliche Dinge optimiert. Steht beim reinen Standortbezug die lokale Verringerung der Umweltbelastung bzw. der Einsatz der lokal benötigten Ressourcen im Vordergrund, so geht es beim Produktbezug um eine Verringerung der Umweltbelastung insgesamt, also ortsunspezifisch und insbesondere umweltmedienübergreifend. Meistens sind beim Produktlebensweg die Umweltbelastungen durch die Bereitstellung von Rohstoffen, die Nutzungsphase beim Verbraucher sowie die Entsorgung von zentraler Bedeutung für die ökologische Beurteilung eines Produktes. Beide Ansätze stehen somit nicht in Konkurrenz zueinander, sondern sie ergänzen sich im Sinne einer Gesamtoptimierung.

Die Dualität der beiden Ansätze spiegelt sich auch in der Methodendiskussion wieder. So existieren in der Ökobilanz-Diskussion sowohl betriebliche Umweltbilanzen, die seit Anfang der 90er Jahre hauptsächlich im deutschsprachigen Raum weit verbreitet sind, als auch produktbe-

\section{Nachbaltige Regional- entwicklung in Europa}

ist das Thema des Informationsdienstes

Ökologisches Wirtschaften 5/98

Wenn Sie potentielle Beiträge haben, wenden Sie sich bitte an die Redaktion! zogene Ökobilanzen, im Englischen als Life Cycle Assessment (LCA) bezeichnet. In der ISONormenreihe 14000 ist LCA als Methodik mit den Normen ISO $14040 \mathrm{ff}$. fest verankert.

Für die Umweltpolitik eines Unternehmens sind beide Perspektiven - Standort und Produkt von Bedeutung. Bei den Produkten ist das Unternehmen z. B. als Akteur in der umweltfreundlichen Auswahl von Werkstoffen, in der ökologischen Optimierung der Nutzungsphase und Entsorgung bereits zum Zeitpunkt der Produktentwicklung oder in der geeigneten Auswahl von Vorlieferanten und Distributionssystemen gefordert. Umweltpolitische Instrumente hierzu sind produktbezogene Umweltzeichen wie z.B. der Blaue Engel in Deutschland oder das europäische Umweltgütezeichen, wodurch der Produktbezug für die Unternehmen auch mit einem Marketingeffekt verbunden sein kann.

\section{Untrennbares getrennt?}

An der EG-Öko-Audit-Verordnung wird nun häufig bemängelt, daß sie weder eine Umweltverträglichkeitsprüfung von Unternehmen noch von Produkten umfasse: ,Was sie produzieren, spielt für das EG-Umwelt-Audit keine Rolle“ (1). Beispielsweise kritisiert Dyllick, daß die Verordnung sich im Kern nur auf die Betriebsstandorte an den Standorten industrieller Produktion beziehe, nicht aber auf die Produkte selbst (2). Damit zertrenne man aber einen Zusammenhang, der sich sachlich nicht zertrennen lasse. Ökologische Belastungen entstünden sowohl bei den standortspezifischen Betriebsprozessen als auch bei den Produkten selber. In einem Umweltmanagementkonzept seien deshalb beide Bereiche, als zentrale ökologische Handlungsfelder zu erfassen und zu regeln.

Diese Kritik ist nur teilweise berechtigt. Die EGÖko-Audit-Verordnung greift, wenngleich nur kursorisch, durchaus verschiedene Aspekte aus dem Verantwortungsbereich der Unternehmenauf. Im Anhang I Abschnitt B Punkt 4 wird unter Aufbau- und Ablaufkontrolle auf den Einfluß des Unternehmens auf Vertragspartner und Lieferanten hingewiesen. Unter Abschnitt $\mathrm{C}$, zu behandelnde Gesichtspunkte“ wird explizit auf die „Produktplanung (Design, Verpackung, Transport, Verwendung und Endlagerung) “ eingegangen. Unter Abschnitt D ,Gute Managementpraktiken“ wird die Forderung erhoben, daß ,die Umweltauswirkungen jeder neuen Tätigkeit, jeden neuen Produkts und jedes neuen Verfahrens [...] im voraus beurteilt [werden]." Die EG-Öko-Audit-Verordnung versucht damit, zumindest theoretisch, einen Brückenschlag zur Produktseite im Umweltmanagement. Es darf aber tatsächlich bezweifelt werden, ob dies in der Praxis hat große Relevanz hat.

\section{In der Praxis nebensächlich}

Eine Unternehmensbefragung im Rahmen des Forschungsvorhabens ,Evaluierung von Umweltmanagementssystemen" für das Umweltbundesamt und das Bundesumweltministerium ergab, daß die weitläufige Annahme, die Produktseite werde im Rahmen der betrieblichen Umweltmanagementsysteme nicht berücksichtigt, so pauschal nicht zutrifft. Zwei Drittel der befragten Unternehmen gaben immerhin an, daß die Produktseite bereits einbezogen wird, was allerdings noch nichts über die Qualität dieser Einbeziehung aussagt. Gemeinhin bedeutet das noch nicht, daß etwas Vergleichbares wie Produkt-Ökobilanzen erstellt werden. Trotzdem wird in einigen Fällen die Produktseite bereits außerhalb des Umwelt-Audit-Verfahrens (z. B. im Rahmen von Responsible Care) behandelt. Von den Unternehmen, die die Umweltwirkungen der Produkte bereits berïcksichtigen, erklärten wiederum zwei Drittel, daß sich daraus auch Umweltschutzziele oder wichtige Umweltschutzmaßnahmen ergaben. Interessant ist dabei, daß fast alle dieser Unternehmen die Frage verneinten, ob diese Ziele oder Maßnahmen auch ohne die explizite Produktbetrachtung, also nur bei reiner Standortperspektive, deutlich geworden wären. Dies stiitzt die eingangs erwähnte Aussage, daß Standort- und Produktperspektive komplementäre, sich ergänzende Betrachtungsweisen sind.

Deutlich wurde außerdem, daß die produktbezogenen Maßnahmen in allen Unternehmen die Zusammenarbeit mit anderen Akteuren, in erster Linie mit Kunden und Lieferanten, betref- 
fen. Dies bekräftigt die Annahme, daß die Berücksichtigung der Produktseite den reinen Standort- oder sogar Organisationsrahmen sprengt und einen Austausch der Akteure längs der Produktlebenswege erfordert.

\section{- Methodisch aufwendig, aber lohnend}

Gerade die Umsetzung der in den ,guten Managementpraktiken" geforderten Beurteilung jeder neuen Tätigkeit, jedes neuen Produktes und jedes neuen Verfahrens im voraus weist auf das eigentliche Problem hin, das die Unternehmen meistens beim Produktbezug haben: Es mangelt an handhabbaren und effizienten Verfahren zur Erfassung und Bewertung der Umweltauswirkungen von Produkten, insbesondere von vielen Produkten. In der Praxis ergeben sich für die Unternehmen schwierige methodische Fragen, z. B. zur medienübergreifenden Bewertung oder zur Systemabgrenzung. Wenn in einem Unternehmen Produkte berïcksichtigt werden, dann meistens nur in den relevanten Fällen.

Demgegenüber ist der Standortbezug schon aufgrund der vorwiegenden Ausrichtung des Umweltrechtes auf den Produktionsstandort für die Unternehmen gewohnt sowie methodisch und hinsichtlich des Aufwandes leicht umsetzbar. Eine Ausweitung der EG-Öko-Audit-Verordnung auf den Produktbereich lehnen viele Unternehmen jedoch als eine Überfrachtung des Systems ab. Besonders groß ist die Befürchtung, damit könnte die Durchfïhrung von aufwendigen Produkt-Ökobilanzen für die Unternehmen obligatorisch werden.

Doch die schwierige Frage nach Aufwand und Methodik darf nicht davon ablenken, daß die
Umweltwirkungen der Produkte in ihrer Nutzungs- und Entsorgungsphase häufig die der Produktionsphase weit übertreffen. Ein Unternehmen, das nur seinen Standort ökologisch optimiert, nicht aber seine Produkte, würde damit eine erhebliche Lücke in seiner Umweltpolitik aufweisen. Dies ist auch der Grund für die Berücksichtigung der Produktseite bereits in der heutigen EG-Öko-Audit-Verordnung.

Von besonderer Bedeutung ist dies bei jenen Unternehmen, die keine wesentlichen Umweltbelastungen am Standort verursachen, sondern hauptsächlich zu mittelbaren Umweltbelastungen durch ihre „Produkte“ beitragen. Dies tritt vor allem im nicht-produzierenden Gewerbe auf, z. B. im Dienstleistungsgewerbe. Beispiele hierfür sind Versicherungen oder Reiseunternehmen. Dieser Aspekt ist besonders bei einigen „,neuen“ Branchen relevant, die nun mit der Erweiterungsverordnung in das EMAS-System aufgenommen wurden.

\section{- Marketingvorteil nur vordergründig}

Bei der Frage, ob der Produktaspekt bei der EGÖko-Audit-Verordnung stärker ausgebaut werden soll, muß man auch davor warnen, leichtfertig auf das Argument des Marketingvorteils durch eine stärkere Einbeziehung der Produkte und damit auf eine höhere Attraktivität von EMAS zu setzen, da dies aus Fairneßgründen eine saubere Produktbilanzierung und -bewertung voraussetzt. Dies ist aber sehr aufwendig und wird bisher meistens nur in Einzelfällen durchgeführt. Dürfte umgekehrt auf Produkten mit der (nicht produktbezogenen) EMAS-Beteiligung geworben werden, so wäre dies das Ende der internationa- len Bemühungen um einen anspruchsvollen Standard für das Ecolabelling oder LCA von Produkten.

\section{Fazit: EMAS als Metasystem für den betrieblichen Umwelt- schutz}

Deshalb empfiehlt es sich nicht, die EG-ÖkoAudit-Verordnung obligatorisch auf die Produktseite auszudehnen, einerseits da hier verschiedene Herangehensweisen miteinander vermischt würden, andererseits weil damit methodische und aufwandsbezogene Probleme das Gemeinschaftssystem erheblich belasten würden. Die Frage der Produktbilanzierung und -bewertung muß, da sie nur unternehmensuibergreifend und lebenswegbezogen beantwortet werden kann, als sehr komplex eingestuft werden, was sich in der internationalen LCA-Diskussion widerspiegelt.

Die EG-Öko-Audit-Verordnung sollte hier eher als Metasystem des betrieblichen Umweltschutzes verstanden werden, das die geeigneten Voraussetzungen schafft, ggf. auch produktbezogene Instrumente qualifiziert einzusetzen. Auch die ISO-Normenreihe 14000 unterscheidet zwischen der Einführung eines Umweltmanagementsystems (ISO 14001) und Instrumenten zur Produktbewertung (der LCA mit ISO 14040 ff.). Letztere können natürlich Bestandteil eines Umweltmanagementsystems sein, sind aber gesondert festgelegt.

Der allgemeine Hinweis in der EG-Öko-AuditVerordnung auf die Berücksichtigung der Produktplanung im Rahmen einer betrieblichen Umweltpolitik erscheint hier erst einmal ausreichend. Was fehlt, sind die Querbezüge zu anderen Instrumenten, bei denen der Produktbezug

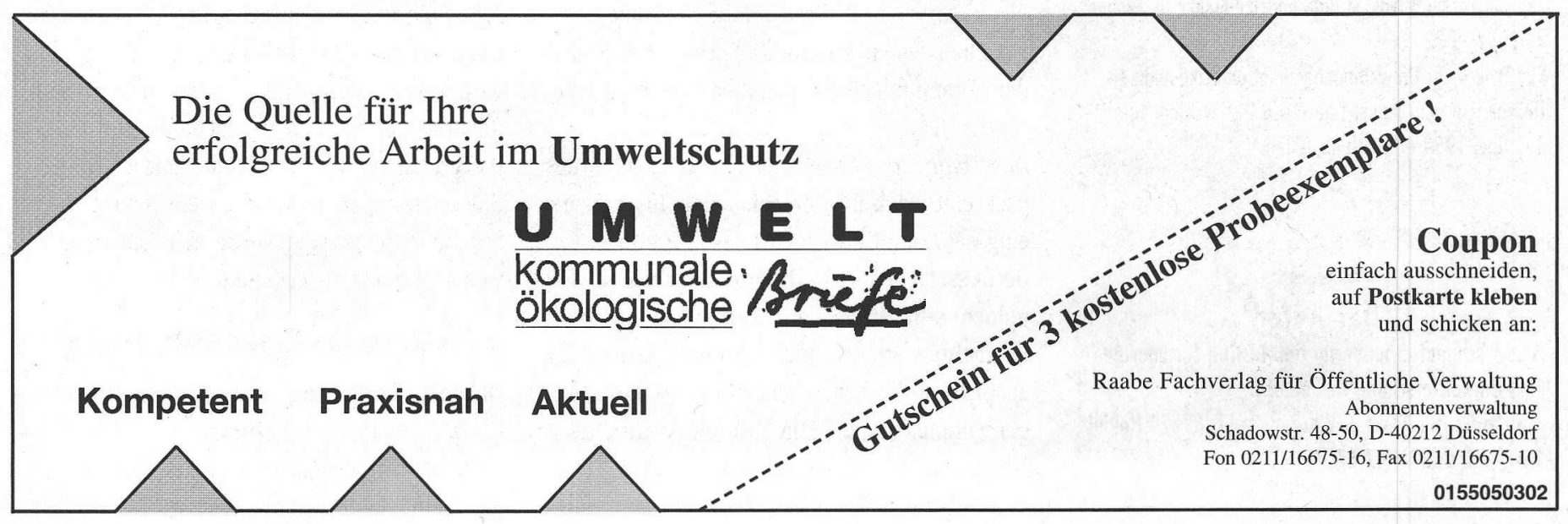


stärker im Vordergrund steht, z. B. den Umweltzeichen. Gerade auf EU-Ebene kann auf das pa-rallel existierende Umweltgütezeichen verwiesen werden. Diese könnten als Optionen oder Anregungen in der Verordnung explizit aufgenommen werden und den Produktaspekt, z. B. im Rahmen der guten Managementpraktiken, konkretisieren.

Auf jeden Fall wäre es wünschenswert, wenn EMAS den durch das Unternehmen bedingten Umweltauswirkungen fernab des Standortes mehr Gewicht beimessen würde. Eine größere Bedeutung könnten in diesem Zusammenhang z.B.

- die unternehmensübergreifende Kooperation,

- die Berïcksichtigung betriebsexterner Umweltbelastungen und

- eine anspruchsvolle Erfassung der Umweltbelastungen, etwa im Sinne einer Umweltbilanz oder Stoffstromanalyse, erhalten. Dies wären sowieso unerläßliche Voraussetzungen für eine stärkere Einbeziehung der Produktseite.

Auch wenn man von konkreten Festlegungen und Verfahren zur Produktseite absieht, so sollte in eine novellierte EG-Öko-Audit-Verordnung doch zumindest folgender Leitsatz in die guten Managementpraktiken für die Umweltpolitik mitaufgenommen werden. Ein Unternehmen muß sich darüber kundig machen, in welchem Handlungsbereich seiner direkten oder indirekten Verantwortung die größten Umweltbelastungen auftreten. Ein Unternehmen sollte dann darüber nachdenken, wie diese Umweltbelastungen verringert werden können und welcher Verfahren man sich dazu bedienen kann.

\section{Anmerkungen}

(1) Glatzner, L.: Was ist dran am Öko-Audit? In: Globus 1/1997, S.33.

(2) Dyllick, T.: Umweltmanagement und Öko-Audit Heilsbringer oder Papier-Tiger? 0̈.B.U./IWÖ-Tagung am 11. Nov. 1994 in Zürich-Altstetten.

\section{Der Autor}

Mario Schmidt arbeitet am ifeu-Institut für Energieund Umweliforschung Heidelberg.

Kontakt: ifeu-Institut, Wilckensstr. 3, 69120 Heidelberg, Tel. 06221/47 67.0.

Ein Vorschlag zur Stärkung des Produktbezugs von EMAS

$$
\text { Step by Step }
$$

\section{Die Umweltpolitik hat sich Produkten zugewendet, wie die Schlagworte Produktverantwortung, Responsible Care oder Lebenszyklusdenken zeigen. Gleichzeitig erhielten "weiche" Instrumente wie EMAS in der Umweltpolitik eine wachsende Bedeutung. Ist im Sinne einer Verknüpfung ein verbesserter Produktbezug von EMAS angezeigt und wie könnte dieser erfolgen?}

$\mathrm{D}$ Von Frieder Rubik ie derzeitige Ausgestaltung von EMAS eröffnet nur wenige Andockstellen zwischen Umweltmanagementsystem und Produktbezug:

- Anhang I C Nr. 7: Beachtung der „Produktplanung (Design, Verpackung, Transport, Verwendung und Endlagerung)" bei Umweltpolitik, -programm und -managementsystem;

- Anhang I C Nr. 8: Berücksichtigung von Auftragnehmern und Lieferanten;

- Anhang I D Nr. 2: „Die Umweltauswirkungen (...) jedes neuen Produkts (...) werden im voraus beurteilt".

Empirische Untersuchungen ergaben, daß der Produktbezug in Umweltmanagementsystemen nach EMAS derzeit nur unzureichend quantitativ und qualitativ realisiert wird (1). Wenn EMAS, wie einige Beiträge in diesem Heft anregen, in Richtung eines ökologischen Gütesiegels ausgebaut würde, dann wäre die Teilnahme und erfolgreiche Registrierung nach EMAS ein Anhaltspunkt für "Cleaner production“. Doch kann daraus dann auch auf "Cleaner products“ geschlossen werden? Die Antwort ist - nach derzeitiger Lage - nein.

Die Frage ist daher, ob die Verstärkung des Produktbezugs von EMAS sinnvoll ist oder nicht. Wenn ja, wie soll diese erfolgen? Zunächst ist zwischen einem innerbetrieblichen Fokus und der Kommunikation gegenüber Dritten zu differenzieren.

Innerhalb von Unternehmen werden bereits jetzt eine Vielzahl verschiedener Instrumente eingesetzt, um Umweltaspekte von Produkten zu berücksichtigen (2). Diese unterscheiden sich jedoch sehr stark nach Formalisierungsgrad, Betrachtungstiefe und -breite. Das sehr anspruchsvolle Instrument der produktbezogenen Ökobilanz nach DIN ISO 14040 wird zweifelsohne nicht flächendeckend eingesetzt. Selbst Unternehmen, bei denen Ökobilanzen „routi- nemäßig" verwendet werden, sind weit davon entfernt, diese bei allen Produktinnovationen heranzuziehen. Hauptgründe sind die vergleichsweise hohen methodischen Anforderungen sowie die aufwendige Datensuche und -zusammenstellung.

In der Kommunikation gegenüber Dritten können Unternehmen eigene Zeichen kreieren, offizielle Umweltzeichen (wie z.B. den „Blauen Engel") verwenden oder quantitative bzw. qualitative Informationen weitergeben.

Diese beiden Ansatzpunkte haben zunächst einmal keinen Bezug zu EMAS. Er stellt sich erst durch den lauter werdenden Wunsch her, die erfolgreiche Teilnahme an EMAS in der Produktwerbung verwenden zu dürfen. Hiervon erhoffen sich Unternehmen einen Nutzen, etwa Imageverbesserung oder Umsatzerhöhung. Aus der Konsumforschung wissen wir, daß VerbraucherInnen für Kaufentscheidungen einfache, eindeutige Zeichen als ,Schlüsselinformationen " heranziehen, denn nur diese reduzieren die Transaktionskosten. Beispiele sind der „Blaue Engel“ oder die „Umweltblume“ der EG. Ein graphisch überarbeitetes EMAS-Symbol könnte als „Schlüsselinformation“ zusätzliche Informationen bereitstellen. Aber ein derartiges Symbol droht seine Glaubwürdigkeit zu verlieren, sofern der schwache Produktbezug von EMAS nicht verändert wird. Folge: Das Symbol wird keine Anerkennung finden. Daraus ist zu folgern, daß unter den gegebenen Umständen die Erlaubnis, die EMAS-Teilnahme in der Produktwerbung zu verwenden, ein Schritt in die falsche Richtung sein würde. Ich sehe dennoch weiterführende Ansatzpunkte.

\section{Integration in Umweltzeichen}

Offizielle Umweltzeichensysteme haben einen schwachen Produktionsbezug; das Schwergewicht der Anforderungskriterien liegt zumeist im Bereich der Produktnutzung und -beseiti- 
(c) 20I0 Authors; licensee IÖW and oekom verlag. This is an article distributed under the terms of the Creative Commons Attribution Non-Commercial No Derivates License (http://creativecommons.org/licenses/by-nc-nd/3.o/), which permits unrestricted use, distribution, and reproduction in any medium, provided the original work is properly cited. 